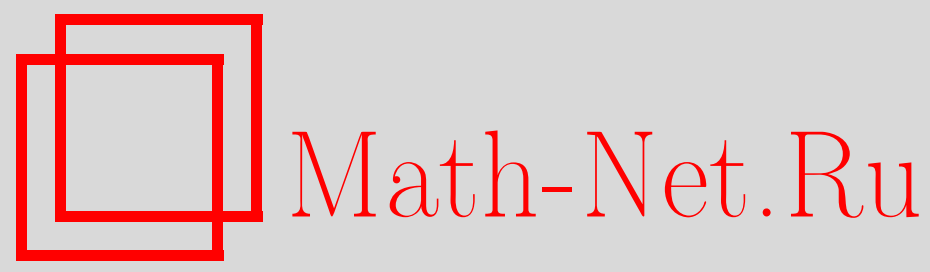

А. С. Печенцов, А. Ю. Попов, Асимптотическое поведение спектральной функции одного семейства операторов, $\mathrm{Ma}$ тем. заметки, 1997, том 61, выпуск 5, 793-796

DOI: https://doi.org/10.4213/mzm1562

Использование Общероссийского математического портала Math-Net.Ru подразумевает, что вы прочитали и согласны с пользовательским соглашением http://www . mathnet.ru/rus/agreement

Параметры загрузки:

IP : 35.174 .16 .151

26 апреля 2023 г., 12:52:52

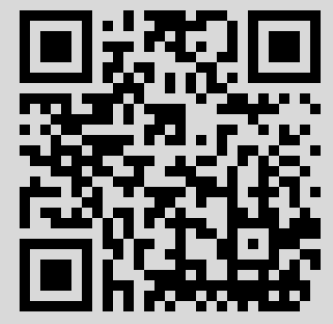




\section{АСИМПТОТИЧЕСКОЕ ПОВЕДЕНИЕ СПЕКТРАЛЬНОЙ ФУНКЦИИ ОДНОГО СЕМЕЙСТВА ОПЕРАТОРОВ}

\section{А. С. Печенцов, А. Ю. Попов}

В $L_{2}[0,+\infty)$ рассмотрим оператор $L$, задаваемый дифференциальньм выражением

$$
l y(x)=-y^{\prime \prime}(x)+q(x) y(x)
$$

и граничным условием

$$
y(0) \cos \alpha+y^{\prime}(0) \sin \alpha=0 .
$$

Предполагается, что $\alpha \in \mathbb{R}, q$ - действительнозначная функция, непрерывная на $[0,+\infty)$. Обозначим через $\varphi(x, \lambda)$ и $\theta(x, \lambda)$ решение уравнения $l y=\lambda y$ с начальньми условиями

$$
\varphi(0, \lambda)=\sin \alpha, \quad \varphi^{\prime}(0, \lambda)=-\cos \alpha, \quad \theta(0, \lambda)=\cos \alpha, \quad \theta^{\prime}(0, \lambda)=\sin \alpha .
$$

Функции $\varphi, \theta$, а также $\rho, m, \widehat{f}$ (они появятся ниже) зависят, естественно, и от $\alpha$, но мы будем опускать аргумент $\alpha$ для того, чтобы не перегружать текст работы обозначениями. Хорошо известна теорема Г. Вейля о представлении произвольной функции $f \in L_{2}[0,+\infty)$ в виде интеграла по спектру оператора $L$.

Теорема [1]-[3]. Существует неубывающая и ограниченная снизу на $\mathbb{R}$ функиия $\rho$, для которой справедливы следующие утверждения.

1. Существует

$$
\widehat{f}(\lambda) \underset{L_{2}(\mathbb{R}, d \rho)}{=} \lim _{n \rightarrow \infty} \int_{0}^{n} f(x) \varphi(x, \lambda) d x=\int_{0}^{+\infty} f(x) \varphi(x, \lambda) d x,
$$

а функиия $f$ представляется как предел в $L_{2}[0,+\infty)$ преобразования Фурье по мере d $\rho$ om $\widehat{f}$ :

$$
f(x) \underset{L_{2}\left(\mathbb{R}^{+}\right)}{=} \lim _{n \rightarrow \infty} \int_{-n}^{n} \widehat{f}(\lambda) \varphi(x, \lambda) d \rho(\lambda)=\int_{-\infty}^{+\infty} \widehat{f}(\lambda) \varphi(x, \lambda) d \rho(\lambda) .
$$

2. Если $\sin \alpha \neq 0$, то для функиии $m(z)$, определяемой в полуплоскости $\operatorname{Im} z>0$ равенством

$$
m(z)=-\operatorname{ctg} \alpha+\int_{-\infty}^{+\infty} \frac{d \rho(\lambda)}{z-\lambda},
$$

имеем $\theta(x, z)+m(z) \varphi(x, z) \in L_{2}[0,+\infty) \forall z: \operatorname{Im} z>0$.

3. Для любого борелевского множества $E \subset \mathbb{R}$, не содержашего спектра оператора $L$, имеем $\int_{E} d \rho(\lambda)=0$.

Утверждение 3 теоремы показьвает, что мера $d \rho$ сосредоточена на спектре опера-

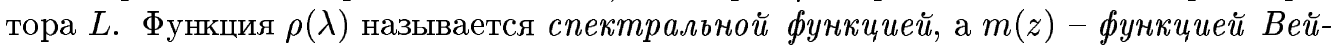
ля-Tитчмарша оператора $L$. Из представления (1) видно, что функция $m(z)$ аналитична в верхней полуплоскости и что если на некотором интервале $(a, b) \subset \mathbb{R}$ существует

$$
\lim _{y \rightarrow+0} m(\lambda+i y)=m(\lambda) \in C(a, b)
$$

(C) А. С. Печенцов, А. Ю. Попов 
то $\rho(\lambda)$ имеет на этом интервале непрерывную производную, которая связана с $m(\lambda)$ соотношением

$$
\rho^{\prime}(\lambda)=\frac{-\operatorname{Im} m(\lambda)}{\pi}, \quad \lambda \in(a, b) .
$$

Исследование свойств функции $p(\lambda)$, а тем более ее отыскание по потенциалу $q$ и числу $\alpha$, определяющим оператор $L$, является, как правило, очень трудной задачей. Известны общие теоремы Б. М. Левитана и В.А. Марченко (см. монографии [4]-[6]) об оценках $\rho(\lambda)$ при $\lambda \rightarrow \pm \infty$ :

$$
\begin{aligned}
& \rho(\lambda)-\rho(-\infty)=o(\exp (-a \sqrt{|\lambda|})) \quad(\lambda \rightarrow-\infty) \quad \forall a>0, \\
& \rho(\lambda)=\frac{2 \sqrt{\lambda}}{\pi \sin ^{2} \alpha}+\rho(-\infty)+\frac{\cos \alpha}{\sin ^{2} \alpha}+\bar{o}(1) \quad(\lambda \rightarrow+\infty) .
\end{aligned}
$$

(Считается, что произведена нормировка $\rho(0)=0$.)

Самьй простой случай $q(x) \equiv 0$ был исследован Титчмаршем в [7]. Оказалось, что при $\lambda>0$

$$
\rho^{\prime}(\lambda)=\frac{\sqrt{\lambda}}{\pi\left(\lambda \sin ^{2} \alpha+\cos ^{2} \alpha\right)},
$$

а при $\lambda<0$ случаи $\operatorname{ctg} \alpha>0$ и $\operatorname{ctg} \alpha<0$ резко различаются:

$$
\begin{gathered}
\rho_{\alpha}^{\prime}(\lambda) \equiv 0, \quad \lambda<0, \quad \operatorname{ctg} \alpha<0, \\
\rho_{\alpha}^{\prime}(\lambda)=\frac{2 \operatorname{ctg} \alpha}{\sin ^{2} \alpha} \delta\left(\lambda-\lambda_{0}\right), \quad \lambda<0, \quad \operatorname{ctg} \alpha>0,
\end{gathered}
$$

где $\lambda_{0}=-\operatorname{ctg}^{2} \alpha, \delta(\cdot)$ - дельта-функция Дирака.

В этой работе мы исследуем случай $\operatorname{tg} \alpha>0$ и интересуемся поведением производной спектральной функции на $(-\infty, 0)$ для потенциалов специального вида.

Вызывает интерес следующая задача общего вида.

Пусть $Q \in C(0,+\infty)$. Через $\rho_{\alpha}(\varepsilon, Q, \lambda)$ обозначим спектральную функцию оператоpa, задаваемого дифференциальным выражением

$$
-y^{\prime \prime}(x)+\varepsilon Q(x) y(x)
$$

и граничным условием

$$
y(0) \cos \alpha+y^{\prime}(0) \sin \alpha=0, \quad \operatorname{ctg} \alpha>0 .
$$

Верно ли, что на $(-\infty, 0)$ семейство обобщенных функций $\rho_{\alpha}^{\prime}(\varepsilon, Q, \lambda)$ сходится при $\varepsilon \rightarrow 0$ (в каком-нибудь "разумном" смысле) к производной спектральной функции (3) невозмущенного оператора $-y^{\prime \prime}$ ? Положительное решение этой задачи позволило бы для функций $f \in L_{2}[0,+\infty)$ писать “приближенное спектральное разложение" в виде

$$
f(x)=\int_{\lambda_{0}-\beta}^{\lambda_{0}+\beta}+\int_{-\beta}^{+\infty} \widehat{f}(\lambda) \varphi_{\alpha}(x, \lambda) d \rho_{\alpha}(\varepsilon, Q, \lambda) \quad(\beta=\beta(\varepsilon) \downarrow 0),
$$

опуская интеграл по дополнению к малой окрестности спектра невозмущенного оператора. Насколько нам известно, такие исследования ранее не проводились. Титчмарш в работе [7] выразил через функции Ганкеля 1-го рода функцию Вейля-Титчмарша $m(\lambda, \varepsilon)$ оператора

$$
-y^{\prime \prime}-\varepsilon x y, \quad \varepsilon>0, \quad y(0) \cos \alpha+y^{\prime}(0) \sin \alpha=0, \quad \operatorname{ctg} \alpha>0 .
$$


Он установил, что при всех $\lambda \in \mathbb{R}$

$$
m(\lambda, \varepsilon)=\frac{H_{1 / 3}^{(1)}(A) \sin \alpha-\sqrt{\lambda} H_{-2 / 3}^{(1)}(A) \cos \alpha}{H_{1 / 3}^{(1)}(A) \cos \alpha+\sqrt{\lambda} H_{-2 / 3}^{(1)}(A) \sin \alpha}
$$

где $A=2 \lambda^{3 / 2} /(3 \varepsilon), \lambda^{3 / 2}=-i|\lambda|^{3 / 2}$ при $\lambda<0, H_{p}^{(1)}-$ функции Ганкеля 1-го рода.

С помошью представления (4) в работе [7] было доказано, что в некоторой окрестности точки $\lambda_{0}$ у функций $m(\lambda, \varepsilon)$ имеется лишш один полюс $\lambda_{0}(\varepsilon)$ с асимптотикой

$$
\lambda_{0}(\varepsilon)=\lambda_{0}-\frac{\varepsilon}{2} \operatorname{tg} \alpha+O\left(\varepsilon^{2}\right) \quad(\varepsilon \rightarrow+0),
$$

а мнимая часть его удовлетворяет неравенству

$$
-\exp \left(-\frac{\operatorname{ctg}^{3} \alpha}{\varepsilon}\right) \leqslant \operatorname{Im} \lambda_{0}(\varepsilon)<0 .
$$

Но поведение $\rho^{\prime}(\lambda, \varepsilon)$ при $\lambda \in(-\infty, 0)$ и $\varepsilon \rightarrow+0$ Титчмарш не исследовал. Нам удалось в определенном смысле почти до конца решить эту задачу. Несмотря на наличие явной формулы для $\rho^{\prime}(\lambda, \varepsilon)$ (ввиду (2) имеем $\rho^{\prime}(\lambda, \varepsilon)=-\operatorname{Im} m(\lambda, \varepsilon) / \pi$, так как из (4) следует, что функция $m(\lambda, \varepsilon)$ аналитична в замкнутой верхней полуплоскости с выброшенной точкой 0) это оказалось далеко не просто. Дело в том, что если подставить в (4) известные асимптотические ряды для $H_{1 / 3}^{(1)}(A)$ и $H_{-2 / 3}^{(1)}(A)$ при $A \rightarrow-i \infty$ (по отрищательной части мнимой оси), то для $m(\lambda, \varepsilon)$ получится асимптотический ряд, все члены которого вещественны. Следовательно, указанньй ряд не дает никакой информации о $\rho^{\prime}(\lambda, \varepsilon)$.

Мы получили следующее представление для функции $\rho^{\prime}(\lambda, \varepsilon)$, справедливое при любых $\lambda<0$ и $\varepsilon>0$ :

$$
\rho^{\prime}(\lambda, \varepsilon)=\frac{\pi^{-1} \tau\left(\beta_{1,1}(a) \beta_{2,2}(a)+\beta_{1,2}(a) \beta_{2,1}(a)\right)}{\left(\beta_{1,1}(a) \cos \alpha-\beta_{1,2}(a) \tau \sin \alpha\right)^{2}+\left(\beta_{2,1}(a) \cos \alpha+\beta_{2,2}(a) \tau \sin \alpha\right)^{2}},
$$

где $\tau=\sqrt{-\lambda}, a=|A|=2 \tau^{3} /(3 \varepsilon)$,

$$
\begin{gathered}
\beta_{1,1}(a)=1+\frac{\sqrt{3}}{2} \frac{B_{1 / 3}^{-}(a)}{\Omega_{1 / 3}(a)}, \quad \beta_{1,2}(a)=\frac{\Omega_{2 / 3}(a)}{\Omega_{1 / 3}(a)}+\frac{\sqrt{3}}{2} \frac{B_{2 / 3}^{-}(a)}{\Omega_{1 / 3}(a)}, \\
\beta_{2,1}(a)=\frac{B_{1 / 3}^{+}(a)}{2 \Omega_{1 / 3}(a)}, \quad \beta_{2,2}(a)=\frac{B_{2 / 3}^{+}(a)}{2 \Omega_{1 / 3}(a)}, \\
\Omega_{p}(a)=\frac{1}{\pi} \int_{0}^{\pi} \exp (a \cos t) \cos (p t) d t, \\
B_{p}^{-}(a)=\frac{1}{\pi} \int_{0}^{+\infty} \exp (-a \operatorname{ch} t) \operatorname{sh}(p t) d t, \quad B_{p}^{+}(a)=\frac{1}{\pi} \int_{0}^{+\infty} \exp (-a \operatorname{ch} t) \operatorname{ch}(p t) d t .
\end{gathered}
$$

Представление (5) позволило доказать формулируемые ниже теоремы 1-3.

ТЕОРемА 1. При любом $\varepsilon>0$ справедлива асимптотика

$$
\rho^{\prime}(\lambda, \varepsilon) \sim \frac{\exp \left(-\frac{4}{3} \frac{|\lambda|^{3 / 2}}{\varepsilon}\right)}{\pi \sqrt{-\lambda} \sin ^{2} \alpha} \quad(\lambda \rightarrow-\infty) .
$$


Теорема 2. СУществуют положительные постоянные $c_{1}$ и $c_{2}$, эффективно зависящие от $\alpha$, такие, что при $\varepsilon \in\left(0, c_{1}\right)$ выполняются оценки

$$
\begin{array}{ll}
\rho^{\prime}(\lambda, \varepsilon)=O\left(|\lambda|^{-1 / 2} \exp \left(-\frac{4|\lambda|^{3 / 2}}{3 \varepsilon}\right)\right), & \lambda \in\left(-\infty,-2 \operatorname{ctg}^{2} \alpha\right), \\
\rho^{\prime}(\lambda, \varepsilon)=O\left(|\lambda|^{1 / 2} \exp \left(-\frac{4|\lambda|^{3 / 2}}{3 \varepsilon}\right)\right), & \lambda \in\left(-\frac{1}{2} \operatorname{ctg}^{2} \alpha,-c_{2} \varepsilon^{2 / 3}\right), \\
\rho^{\prime}(\lambda, \varepsilon)=O\left(\varepsilon^{1 / 3}\right), & \lambda \in\left[-c_{2} \varepsilon^{2 / 3}, 0\right) .
\end{array}
$$

Постоянные в символах $O$ зависят только от $\alpha$ и әффективны.

Обозначим $T(\lambda, \varepsilon)=\beta_{1,1}(a) \cos \alpha-\beta_{1,2}(a) \tau \sin \alpha$. Ответ на вопрос о поведении $\rho^{\prime}(\lambda, \varepsilon)$ на "критическом" отрезке $I=\left[-2 \operatorname{ctg}^{2} \alpha,-\frac{1}{2} \operatorname{ctg}^{2} \alpha\right]$ дает

Теорема 3. Существует полохительная постоянная $c_{3}$, әффективно зависящая от $\alpha$, такая, что при любых $\varepsilon \in\left(0, c_{3}\right)$ справедливы следующие утверэддения.

1. Функция $T(\lambda, \varepsilon)$ имеет на отрезке I единственный нуль $\lambda_{1}(\varepsilon)$ с асимптотикой

$$
\lambda_{1}(\varepsilon)=\lambda_{0}-\frac{\varepsilon}{2} \operatorname{tg} \alpha+O\left(\varepsilon^{2}\right) .
$$

2. При любых $d>0, \lambda \in I \backslash I_{d, \varepsilon}\left(I_{d, \varepsilon}=\left[\lambda_{1}(\varepsilon)-d, \lambda_{1}(\varepsilon)+d\right]\right)$ выполняется оченка

$$
\rho^{\prime}(\lambda, \varepsilon)=O\left(d^{-2} \exp \left(-\frac{4|\lambda|^{3 / 2}}{3 \varepsilon}\right)\right) .
$$

3. $E c л u$

$$
\exp \left(-\frac{\operatorname{ctg}^{3} \alpha}{3 \varepsilon}\right) \leqslant d \leqslant \varepsilon^{2},
$$

mo

$$
\int_{I_{d, \varepsilon}} d \rho(\lambda)=\frac{2 \operatorname{ctg} \alpha}{\sin ^{2} \alpha}+O(\varepsilon) .
$$

СлЕдСтвиЕ. В пространстве $C^{*}(I)$ семейство функций $\rho^{\prime}(\lambda, \varepsilon)$ слабо сходит$\cos \kappa$

$$
\frac{2 \operatorname{ctg} \alpha}{\sin ^{2} \alpha} \delta\left(\lambda-\lambda_{0}\right)
$$

npu $\varepsilon \rightarrow+0$.

Московский государственный университет

Поступило

им. М.В. Ломоносова

09.12 .96

E-mail: pech@imiss.math.msu.su

\section{СПИСОК ЦИТИРОВАННОЙ ЛИТЕРАТУРЫ}

1. Weyl H. // Math. Ann. 1910. V. 68. P. 220-269. 2. Weyl H. // Nachr. Akad. Wiss. Göttingen. Math.-Phys. Kl. 1909. P. 37-64. 3. Weyl H. // Nachr. Akad. Wiss. Göttingen. Math.-Phys. Kl. 1910. Р. 442-467. 4. Левитан Б. М., Саргсян И. С. Введение в спектральную теорию. М.: Наука, 1970. 5. Левитан Б. М. Обратные задачи Штурма-Лиувилля. М.: Наука, 1984. 6. Марченко В. А. Операторы Штурма-Лиувилля и их приложения. Киев: Наукова думка, 1977. 7. Titchmarsh E. C. // Proc. Roy. Soc. London. Ser. A. 1951. V. 207. P. 321-328. 